\title{
PENNY-SHAPED CRACK BRIDGED BY FIBRES
}

\author{
$\mathrm{BY}$ \\ N. V. MOVCHAN (School of Mathematical Sciences, University of Bath, U.K.) \\ AND \\ J. R. WILlIS (Department of Applied Mathematics and Theoretical Physics, University of \\ Cambridge, U.K.)
}

\begin{abstract}
An axi-symmetric problem for a penny-shaped crack bridged by fibres is considered. The study is reduced to the analysis of a hypersingular integral equation with respect to the relative crack-face separation over a circular domain occupied by the crack. The special case of a large crack subjected to Mode-I loading is examined. A matched asymptotic expansions technique is used in order to estimate the stress intensity factor at the crack edge and to evaluate the magnitude of the applied load which provides the critical opening for failure of fibres at the centre of the crack.
\end{abstract}

Introduction. The physical problem that motivated this study relates to a body composed of a brittle matrix (in practice, a ceramic) reinforced by parallel fibres. The matrix contains a circular crack, in a plane normal to the axes of the fibres. The fibres, however, remain intact. They "bridge" the crack and carry some load, thereby increasing the material's resistance to catastrophic failure. The simplest model for this system represents the intact composite as an elastic continuum and the effect of the bridging fibres is taken into account by a continuous distribution of (generally nonlinear) springs, joining opposite points on the crack faces. Application of the model requires two steps: the determination of an appropriate force-separation law that characterizes the restraining tractions provided by the fibres, and stress analysis performed for a crack in a body loaded in some prescribed way at points remote from the crack. In the present paper we deal only with the second step, namely we consider a penny-shaped bridged crack in an elastic infinite body and describe the effect of fibres by introducing restraining tractions that follow either a linear or a quadratic force-separation law.

The problem is formulated in terms of a hypersingular integral equation over a domain occupied by the crack. In general, this can be solved numerically [1], [2]. In the particular case of a long crack, the governing equation contains a small parameter that multiplies the hypersingular integral, leading to a singular perturbation problem. A method for

Received August 17, 1995.

1991 Mathematics Subject Classification. Primary 73C05. 
systematic treatment of such problems was presented in [3], in which explicit results were obtained for the corresponding problem for a long straight crack subjected to a Mode-I loading in plane strain, assuming a linear force-separation law for the bridging fibres. Results for the same configuration but with a particular nonlinear force-separation law were given in [4].

The purpose of the present paper is to extend the method [3] to the case of a pennyshaped bridged crack and to evaluate the stress intensity factor at the crack edge and the magnitude of the applied load that provides the critical opening at the centre of the crack. Two critical conditions, for matrix crack growth with fibres remaining intact and for fibre failure preceding crack extension, are analyzed.

Formulation of the problem. A circular crack occupying the region

$$
S=\left\{\left(\bar{x}_{1}, \bar{x}_{2}, \bar{x}_{3}\right): \bar{x}_{3}=0,\|\overline{\mathbf{x}}\|<a\right\}
$$

where $a>0$ and $\overline{\mathbf{x}}=\left(\bar{x}_{1}, \bar{x}_{2}\right)$, is situated in an otherwise intact infinite body. Loading remote from the crack generates a uniform state of stress in which only the tensile component $\bar{\sigma}_{33} \equiv \bar{\sigma}$ differs from zero. The perturbation of this field produced by the crack can be analyzed in terms of the relative displacement $\overline{\mathbf{b}}$ of the upper and lower faces of the crack. By symmetry, only the third component of $\overline{\mathbf{b}}$ differs from zero, and this is a function of $\|\overline{\mathbf{x}}\|$ only:

$$
\overline{\mathbf{b}}=(0,0, \bar{b}(\|\overline{\mathbf{x}}\|))
$$

Assume that the force-separation law has the form

$$
\bar{\gamma}=\bar{\gamma}(\bar{b}(\|\overline{\mathbf{x}}\|)) \text {. }
$$

In this case the problem can be formulated as an integral equation with respect to the function $\bar{b}(\|\overline{\mathbf{x}}\|)$,

$$
\int_{S} d s \bar{b}(\|\overline{\mathbf{x}}\|) \Gamma\left(\overline{\mathbf{x}}, \overline{\mathbf{x}}^{\prime}\right)+\bar{\gamma}\left(\bar{b}\left(\left\|\overline{\mathbf{x}}^{\prime}\right\|\right)\right)=\bar{\sigma}, \quad\left\|\overline{\mathbf{x}}^{\prime}\right\|<a
$$

where $\bar{b}$ vanishes on the boundary $\partial S$ of the region $S$. Here

$$
\Gamma\left(\overline{\mathbf{x}}, \overline{\mathbf{x}}^{\prime}\right)=-\frac{\mu(\lambda+\mu)}{2 \pi(\lambda+2 \mu)} \frac{1}{\left\|\overline{\mathbf{x}}-\overline{\mathbf{x}}^{\prime}\right\|^{3}}
$$

and $\lambda, \mu$ are the Lamé moduli. ${ }^{1}$

Introduce a system of polar coordinates $(\bar{r}, \theta)$ with the origin at the centre of the disk $S$. In these coordinates, Eq. (1) takes the form

$$
-\frac{\mu(\lambda+\mu)}{2 \pi(\lambda+2 \mu)} \int_{0}^{a} \int_{0}^{2 \pi} \frac{\bar{r} \bar{b}(\bar{r}) d \bar{r} d \theta}{\left(\bar{r}^{2}+\left(\bar{r}^{\prime}\right)^{2}-2 \bar{r}^{\prime} \bar{r} \cos \left(\theta-\theta^{\prime}\right)\right)^{3 / 2}}+\bar{\gamma}\left(\bar{b}\left(\bar{r}^{\prime}\right)\right)=\bar{\sigma}, \quad\left(\bar{r}^{\prime}, \theta^{\prime}\right) \in S .
$$

\footnotetext{
${ }^{1}$ The body could equally well be modelled as transversely isotropic with axis of symmetry parallel to the fibres. The form of the kernel $\Gamma\left(\overline{\mathbf{x}}, \overline{\mathbf{x}}^{\prime}\right)$ would be unchanged except that the constant factor would be replaced by the appropriate combination of the moduli of the transversely isotropic solid.
} 
Integrating with respect to $\theta$ we derive

$$
-\frac{\mu(\mu+\lambda)}{2 \pi(\lambda+2 \mu)} \int_{0}^{a} \frac{\bar{b}(\bar{r})}{\left(\bar{r}-\bar{r}^{\prime}\right)^{2}} \frac{2 \bar{r}}{\bar{r}+\bar{r}^{\prime}} E\left(\frac{2 \sqrt{\bar{r} \bar{r}^{\prime}}}{\bar{r}+\bar{r}^{\prime}}\right) d \bar{r}+\bar{\gamma}\left(\bar{b}\left(\bar{r}^{\prime}\right)\right)=\bar{\sigma}, \quad 0<\bar{r}^{\prime}<a
$$

where $E(k)$ denotes the complete elliptic integral of the second kind (note that Eq. (2) agrees with Eq. (2.9) of [5], if the applied loading is identified with $\left.\bar{\sigma}-\bar{\gamma}\left(\bar{b}\left(\bar{r}^{\prime}\right)\right)\right)$. The solution of (2) is required to satisfy the conditions

$$
\bar{b}(a)=0, \quad \bar{b}^{\prime}(0)=0 .
$$

The integral on the left-hand side of (2) is specified in the finite-part Hadamard sense [6]. As $\bar{r} \rightarrow \bar{r}^{\prime}$, the kernel

$$
\mathcal{K}\left(\bar{r}, \bar{r}^{\prime}\right)=\frac{1}{\left(\bar{r}-\bar{r}^{\prime}\right)^{2}} \frac{2 \bar{r}}{\bar{r}+\bar{r}^{\prime}} E\left(\frac{2 \sqrt{\bar{r} \bar{r}^{\prime}}}{\bar{r}+\bar{r}^{\prime}}\right)
$$

of the integral operator (2) has the asymptotic form

$$
\mathcal{K}\left(\bar{r}, \bar{r}^{\prime}\right) \sim \frac{1}{\left(\bar{r}-\bar{r}^{\prime}\right)^{2}}+\frac{\bar{r}}{\left(\bar{r}+\bar{r}^{\prime}\right)^{3}}\left[2 \ln 2-\ln \left|\bar{r}-\bar{r}^{\prime}\right|+\ln \left(\bar{r}+\bar{r}^{\prime}\right)\right], \quad \bar{r} \rightarrow \bar{r}^{\prime}
$$

As in [4], [7], one can introduce nondimensional variables

$$
\begin{gathered}
\sigma=\bar{\sigma} / \bar{\sigma}_{0}, \quad \gamma=\bar{\gamma} / \bar{\sigma}_{0}, \quad r=\bar{r} / a \\
b=\frac{\mu(\mu+\lambda) \bar{\sigma}_{0} \bar{b}}{2 \pi(\lambda+2 \mu) \bar{K}_{0}^{2}}, \quad \varepsilon=\frac{\bar{K}_{0}^{2}}{\bar{\sigma}_{0}^{2} a}
\end{gathered}
$$

with $\bar{\sigma}_{0}$ taken as some stress characteristic of the force-separation law, such as $\bar{\gamma}^{\prime}(0)$ or the maximum value of $\bar{\gamma}$ if this exists, and the parameter $\bar{K}_{0}$ characterizing the fracture toughness of the unreinforced material, and rewrite the problem (2), (3) in the form

$$
\begin{gathered}
-\varepsilon \int_{0}^{1} \frac{b(r)}{\left(r-r^{\prime}\right)^{2}} \frac{2 r}{\left(r+r^{\prime}\right)} E\left(\frac{2 \sqrt{r r^{\prime}}}{r+r^{\prime}}\right) d r+\gamma\left(b\left(r^{\prime}\right)\right)=\sigma, \quad 0<r^{\prime}<1, \\
b(1)=0, \quad b^{\prime}(0)=0 .
\end{gathered}
$$

We shall construct the asymptotic approximation of the solution of (6), (7) under the assumption that the parameter $\varepsilon$ is small (the case of a penny-shaped crack of large radius, $\left.a \gg\left(\bar{K}_{0} / \bar{\sigma}_{0}\right)^{2}\right)$. We shall follow the asymptotic algorithm developed in [3]. The hypersingular integral on the left-hand side of (7) will be treated with the use of the 
formula

$$
\begin{aligned}
& \int_{0}^{1} \frac{b(r)}{\left(r-r^{\prime}\right)^{2}} \frac{2 r}{\left(r+r^{\prime}\right)} E\left(\frac{2 \sqrt{r r^{\prime}}}{r+r^{\prime}}\right) d r \\
& =\int_{0}^{1} d r\left\{\frac{2 r E\left(\frac{2 \sqrt{r r^{\prime}}}{r+r^{\prime}}\right)}{\left(r-r^{\prime}\right)^{2}\left(r+r^{\prime}\right)}\left[b(r)-b\left(r^{\prime}\right)-\left(r-r^{\prime}\right) b^{\prime}\left(r^{\prime}\right)\right]\right. \\
& \left.\quad-\frac{r^{\prime} b\left(r^{\prime}\right)}{r^{\prime}\left(r+r^{\prime}\right)}\left[E\left(\frac{2 \sqrt{r r^{\prime}}}{r+r^{\prime}}\right)-K\left(\frac{2 \sqrt{r r^{\prime}}}{r+r^{\prime}}\right)\right]\right\} \\
& -b\left(r^{\prime}\right)\left[\frac{1}{1-r^{\prime}} E\left(\frac{2 \sqrt{r^{\prime}}}{1+r^{\prime}}\right)+\frac{1}{1+r^{\prime}} K\left(\frac{2 \sqrt{r^{\prime}}}{1+r^{\prime}}\right)\right] \\
& +\frac{b^{\prime}\left(r^{\prime}\right)}{r^{\prime}}\left(1+r^{\prime}\right)\left[E\left(\frac{2 \sqrt{r^{\prime}}}{1+r^{\prime}}\right)-K\left(\frac{2 \sqrt{r^{\prime}}}{1+r^{\prime}}\right)\right]+\frac{2 b^{\prime}\left(r^{\prime}\right)}{1+r^{\prime}} K\left(\frac{2 \sqrt{r^{\prime}}}{1+r^{\prime}}\right)
\end{aligned}
$$

for any fixed point $r^{\prime}$ of the segment $(0,1)$ at which the function $b$ is differentiable. The representation (8) is obtained in the same way as in [8]: the equalities

$$
\begin{aligned}
\Gamma\left(\mathbf{x}, \mathbf{x}^{\prime}\right)= & \Delta \Xi, \quad \Xi=-\frac{\mu(\lambda+\mu)}{2 \pi(\lambda+2 \mu)} \frac{1}{\left\|\mathbf{x}-\mathbf{x}^{\prime}\right\|}, \\
b(\|\mathbf{x}\|)= & \left\{b(\|\mathbf{x}\|)-b\left(\left\|\mathbf{x}^{\prime}\right\|\right)-\left(\mathbf{x}-\mathbf{x}^{\prime}\right) \cdot \nabla b\left(\left\|\mathbf{x}^{\prime}\right\|\right)\right\} \\
& +\left\{b\left(\left\|\mathbf{x}^{\prime}\right\|\right)+\left(\mathbf{x}-\mathbf{x}^{\prime}\right) \cdot \nabla b\left(\left\|\mathbf{x}^{\prime}\right\|\right)\right\},
\end{aligned}
$$

and a formal application of Green's theorem give the formula

$$
\begin{aligned}
\int_{S_{1}} d s & b(\|\mathbf{x}\|) \Gamma\left(\mathbf{x}, \mathbf{x}^{\prime}\right) \\
= & \int_{S_{1}} d s \Gamma\left(\mathbf{x}, \mathbf{x}^{\prime}\right)\left\{b(\|\mathbf{x}\|)-b\left(\left\|\mathbf{x}^{\prime}\right\|\right)-\left(\mathbf{x}-\mathbf{x}^{\prime}\right) \cdot \nabla b\left(\left\|\mathbf{x}^{\prime}\right\|\right)\right\} \\
& +\int_{\partial S_{1}} d l\left[\frac{\partial \Xi}{\partial n}\left(b\left(\left\|\mathbf{x}^{\prime}\right\|\right)+\left(\mathbf{x}-\mathbf{x}^{\prime}\right) \cdot \nabla b\left(\left\|\mathbf{x}^{\prime}\right\|\right)\right)-\Xi \mathbf{n} \cdot \nabla b\left(\left\|\mathbf{x}^{\prime}\right\|\right)\right]
\end{aligned}
$$

which after the integration with respect to $\theta$ takes the form (8). Here, $\Delta=\partial^{2} / \partial x_{1}^{2}+$ $\partial^{2} / \partial x_{2}^{2}, \nabla=\left(\partial / \partial x_{1}, \partial / \partial x_{2}\right), \mathbf{n}=\left(n_{1}, n_{2}\right)$ is the unit outward normal vector with respect to $\partial S_{1}, S_{1}=\left\{\left(x_{1}, x_{2}\right): x_{1}^{2}+x_{2}^{2} \leq 1\right\}$.

Asymptotic procedure. Suppose that $b\left(r^{\prime}\right)$ admits the asymptotic representations

$$
b\left(r^{\prime}\right) \sim b_{0}\left(r^{\prime}\right)+b_{1}\left(r^{\prime}\right)+\cdots+b_{n}\left(r^{\prime}\right)+o\left(\varepsilon^{n}\right) \quad \text { as } \varepsilon \rightarrow 0,
$$

with $r^{\prime}$ being fixed and different from 1, and

$$
b\left(1-\varepsilon \rho^{\prime}\right) \sim \tilde{b}_{0}\left(\rho^{\prime}\right)+\tilde{b}_{1}\left(\rho^{\prime}\right)+\cdots+\tilde{b}_{m}\left(\rho^{\prime}\right)+o\left(\varepsilon^{m}\right) \quad \text { as } \varepsilon \rightarrow 0,
$$


with fixed $\rho^{\prime}=\frac{1-r^{\prime}}{\varepsilon}$. The functions $b_{i}\left(r^{\prime}\right), \tilde{b}_{i}\left(\rho^{\prime}\right)$ depend also on $\varepsilon$ and $\ln \varepsilon(\ln \varepsilon$ is regarded as $O(1))$. The asymptotic matching principle of Van Dyke [9] gives

$$
\sum_{k=0}^{n} S_{k}^{m}\left(\frac{1-r^{\prime}}{\varepsilon}\right)=\sum_{k=0}^{m} \widetilde{S}_{k}^{n}\left(r^{\prime}\right)
$$

where functions $S_{k}^{m}\left(\rho^{\prime}\right), \widetilde{S}_{k}^{n}\left(r^{\prime}\right)$ are introduced in the following way:

$$
b_{k}\left(1-\varepsilon \rho^{\prime}\right) \sim S_{k}^{m}\left(\rho^{\prime}\right)+o\left(\varepsilon^{m}\right), \quad \tilde{b}_{k}\left(\frac{1-r^{\prime}}{\varepsilon}\right) \sim \widetilde{S}_{k}^{n}\left(r^{\prime}\right)+o\left(\varepsilon^{n}\right) \quad \text { as } \varepsilon \rightarrow 0 .
$$

In this case a formal uniform expansion of $b\left(r^{\prime}\right)$ is specified by

$$
b\left(r^{\prime}\right) \sim \sum_{k=0}^{n}\left(b_{k}\left(r^{\prime}\right)+\tilde{b}_{k}\left(\rho^{\prime}\right)-S_{k}^{n}\left(\rho^{\prime}\right)\right)+o\left(\varepsilon^{n}\right) .
$$

Solution to lowest order. As $\varepsilon \rightarrow 0$ in (6) with $r^{\prime}$ fixed, (6) reduces to the algebraic equation

$$
\gamma\left(b_{0}\right)=\sigma
$$

The solution of this equation is constant; it violates the boundary condition $b_{0}(1)=0$. In order to compensate the discrepancy, we construct a boundary layer approximation $\tilde{b}_{0}\left(\rho^{\prime}\right)$ in the vicinity of the edge of the crack. Since $b_{0}$ is constant, it follows immediately that

$$
S_{0}^{0}\left(\rho^{\prime}\right)=b_{0} .
$$

Now we identify $r^{\prime}$ with $1-\varepsilon \rho^{\prime}$ and expand (6) to lowest order as $\varepsilon \rightarrow 0$, with $\rho^{\prime}$ fixed. Equation (6) is then written in the form

$$
\begin{aligned}
-\varepsilon \int_{0}^{1} & \frac{d r}{\left(r-1+\varepsilon \rho^{\prime}\right)^{2}} \frac{2 r}{r+1-\varepsilon \rho^{\prime}} E\left(\frac{2 \sqrt{r\left(1-\varepsilon \rho^{\prime}\right)}}{r+1-\varepsilon \rho^{\prime}}\right) \\
& \times\left[b_{0}+\tilde{b}_{0}(\rho)-S_{0}^{0}(\rho)\right]+\gamma\left(\tilde{b}_{0}\left(\rho^{\prime}\right)\right)=\sigma .
\end{aligned}
$$

Since $b_{0}-S_{0}^{0}\left(\rho^{\prime}\right)=0$, the integral on the left-hand side of (13) contains only $\tilde{b}_{0}(\rho)$. The transformation $r=1-\varepsilon \rho$ gives

$$
\begin{aligned}
& -\varepsilon \int_{0}^{1} \frac{d r}{\left(r-1+\varepsilon \rho^{\prime}\right)^{2}} \frac{2 r}{r+1-\varepsilon \rho^{\prime}} E\left(\frac{2 \sqrt{r\left(1-\varepsilon \rho^{\prime}\right)}}{r+1-\varepsilon \rho^{\prime}}\right) \tilde{b}_{0}(\rho) \\
& \quad=-\int_{0}^{1 / \varepsilon} \frac{d r}{\left(\rho-\rho^{\prime}\right)^{2}} \frac{2(1-\varepsilon \rho)}{2-\varepsilon \rho-\varepsilon \rho^{\prime}} E\left(\frac{2 \sqrt{(1-\varepsilon \rho)\left(1-\varepsilon \rho^{\prime}\right)}}{2-\varepsilon \rho-\varepsilon \rho^{\prime}}\right) \tilde{b}_{0}(\rho) .
\end{aligned}
$$

The integral on the right-hand side of (14) can be expressed as follows:

$$
\begin{gathered}
-\int_{0}^{1 / \varepsilon} \frac{d \rho}{\left(\rho-\rho^{\prime}\right)^{2}} \frac{2(1-\varepsilon \rho)}{2-\varepsilon \rho-\varepsilon \rho^{\prime}} E\left(\frac{2 \sqrt{(1-\varepsilon \rho)\left(1-\varepsilon \rho^{\prime}\right)}}{2-\varepsilon \rho-\varepsilon \rho^{\prime}}\right) \tilde{b}_{0}(\rho) \\
=-\int_{0}^{\infty} \frac{d \rho}{\left(\rho-\rho^{\prime}\right)^{2}} \tilde{b}_{0}(\rho)+\int_{1 / \varepsilon}^{\infty} \frac{d \rho}{\left(\rho-\rho^{\prime}\right)^{2}} \tilde{b}_{0}(\rho)+O(\varepsilon) .
\end{gathered}
$$


When $\rho>1 / \varepsilon, \tilde{b}_{0}(\rho)$ can be replaced by $\widetilde{S}_{0}^{0}\left(1-\varepsilon \rho^{\prime}\right)$, and due to the asymptotic matching principle (9) it equals $b_{0}$. The evaluation of the second integral on the right-hand side of (15) therefore gives

$$
\begin{aligned}
& -\varepsilon \int_{0}^{1} \frac{d r}{\left(r-1+\varepsilon \rho^{\prime}\right)^{2}} \frac{2 r}{r+1-\varepsilon \rho^{\prime}} E\left(\frac{2 \sqrt{r\left(1-\varepsilon \rho^{\prime}\right)}}{r+1-\varepsilon \rho^{\prime}}\right) \tilde{b}_{0}(\rho) \\
& \quad=-\int_{0}^{\infty} \frac{d \rho}{\left(\rho-\rho^{\prime}\right)^{2}} \tilde{b}_{0}(\rho)+O(\varepsilon) .
\end{aligned}
$$

Thus, the equation for the boundary layer approximation $\tilde{b}_{0}(\rho)$ takes the form

$$
-\int_{0}^{\infty} \frac{d \rho}{\left(\rho-\rho^{\prime}\right)^{2}} \tilde{b}_{0}(\rho)+\gamma\left(\tilde{b}_{0}\left(\rho^{\prime}\right)\right)=\sigma .
$$

The solution of this equation is required to satisfy the conditions

$$
\tilde{b}_{0}(0)=0, \quad \tilde{b}_{0}\left(\rho^{\prime}\right) \sim b_{0} \quad \text { as } \rho^{\prime} \rightarrow \infty .
$$

The problem (16)-(17) is the same as for the case of a two-dimensional plane strain crack. The explicit form of the solution in the case of a linear function $\gamma$ was derived in [3]. In the case of the quadratic force law this problem was studied in [4].

The first-order approximation of $\tilde{b}_{0}\left(\frac{1-r^{\prime}}{\varepsilon}\right)$ as $\varepsilon \rightarrow 0$ is

$$
\tilde{b}_{0}\left(\frac{1-r^{\prime}}{\varepsilon}\right) \sim b_{0}-\frac{\varepsilon b_{0}}{\gamma^{\prime}\left(b_{0}\right)} \frac{1}{1-r^{\prime}}+o(\varepsilon) \equiv \widetilde{S}_{0}^{1}\left(r^{\prime}\right)+o(\varepsilon) .
$$

Solution to first order. The term $b_{1}\left(r^{\prime}\right)$ of the asymptotic expansion of the crack opening displacement satisfies the equation

$$
-\varepsilon \int_{0}^{1} \frac{b_{0}}{\left(r-r^{\prime}\right)^{2}} \frac{2 r}{r+r^{\prime}} E\left(\frac{2 \sqrt{r r^{\prime}}}{r+r^{\prime}}\right) d r+b_{1}\left(r^{\prime}\right) \gamma^{\prime}\left(b_{0}\right)=0
$$

with fixed $r^{\prime}$ in the interval $(0,1)$.

According to the representation (8), the solution $b_{1}$ of Eq. (19) has the form

$$
\begin{gathered}
b_{1}\left(r^{\prime}\right)=-\frac{\varepsilon b_{0}}{\gamma^{\prime}\left(b_{0}\right)}\left[\frac{1}{1-r^{\prime}} E\left(\frac{2 \sqrt{r^{\prime}}}{1+r^{\prime}}\right)+\frac{1}{1+r^{\prime}} K\left(\frac{2 \sqrt{r^{\prime}}}{1+r^{\prime}}\right)\right], \\
b_{1}^{\prime}\left(r^{\prime}\right) \sim-\frac{3}{2} \pi r^{\prime} \frac{\varepsilon b_{0}}{\gamma^{\prime}\left(b_{0}\right)} \quad \text { as } r^{\prime} \rightarrow 0,
\end{gathered}
$$

and, therefore, it satisfies the condition $b_{1}^{\prime}(0)=0$. In $(20), E(k)$ and $K(k)$ are the complete elliptic integrals of the second and first kind, respectively.

Near the edge of the crack $b_{1}$ admits the asymptotic representation

$$
b_{1}\left(1-\varepsilon \rho^{\prime}\right) \sim \frac{b_{0}}{\gamma^{\prime}\left(b_{0}\right)}\left[-\frac{1}{\rho^{\prime}}+\frac{\varepsilon}{2} \ln \varepsilon+\frac{\varepsilon}{2} \ln \rho^{\prime}-\varepsilon+o(\varepsilon)\right] \quad \text { as } \varepsilon \rightarrow 0 .
$$

It violates the boundary condition $b_{1}(1)=0$, and the discrepancy is compensated by the solution of the boundary layer type $\tilde{b}_{1}\left(\rho^{\prime}\right)-S_{1}^{1}\left(\rho^{\prime}\right)$, with

$$
S_{1}^{1}\left(\rho^{\prime}\right)=\frac{b_{0}}{\gamma^{\prime}\left(b_{0}\right)}\left[-\frac{1}{\rho^{\prime}}+\frac{\varepsilon}{2} \ln \varepsilon+\frac{\varepsilon}{2} \ln \rho^{\prime}-\varepsilon\right] .
$$


Due to the asymptotic matching principle (9)

$$
\tilde{b}_{1}\left(\frac{1-r^{\prime}}{\varepsilon}\right) \sim \frac{b_{0}}{\gamma^{\prime}\left(b_{0}\right)}\left[\frac{\varepsilon}{2} \ln \left(1-r^{\prime}\right)-\varepsilon\right] \quad \text { as } \varepsilon \rightarrow 0 .
$$

To obtain an equation for $\tilde{b}_{1}\left(\rho^{\prime}\right)$, we put $r^{\prime}=1-\varepsilon \rho^{\prime}$ in $(6)$ and collect terms up to order $\varepsilon$. Thus,

$$
\begin{aligned}
-\varepsilon \int_{0}^{1} \frac{d r}{\left(r-1+\varepsilon \rho^{\prime}\right)^{2}} \frac{2 r}{r+1-\varepsilon \rho^{\prime}} E\left(\frac{2 \sqrt{r\left(1-\varepsilon \rho^{\prime}\right)}}{r+1-\varepsilon \rho^{\prime}}\right)\left[b_{0}+\tilde{b}_{0}(\rho)\right. \\
\\
\left.\quad-S_{0}^{0}(\rho)+b_{1}(r)+\tilde{b}_{1}(\rho)-S_{1}^{1}(\rho)\right]+\gamma\left(\tilde{b}_{0}\left(\rho^{\prime}\right)+\tilde{b}_{1}\left(\rho^{\prime}\right)\right)=0 .
\end{aligned}
$$

Note that

$$
\begin{aligned}
-\varepsilon \int_{0}^{1} \frac{d r}{\left(r-1+\varepsilon \rho^{\prime}\right)^{2}} \frac{2 r}{r+1-\varepsilon \rho^{\prime}} E\left(\frac{2 \sqrt{r\left(1-\varepsilon \rho^{\prime}\right)}}{r+1-\varepsilon \rho^{\prime}}\right) \\
\times\left[b_{0}-S_{0}^{0}(\rho)+b_{1}(r)-S_{1}^{1}(\rho)\right]=o(\varepsilon),
\end{aligned}
$$

and the integral containing $\tilde{b}_{0}(\rho)$ and $\tilde{b}_{1}(\rho)$ can be written in the form

$$
\begin{aligned}
-\varepsilon \int_{0}^{1} & \frac{d r}{\left(r-1+\varepsilon \rho^{\prime}\right)^{2}} \frac{2 r}{r+1-\varepsilon \rho^{\prime}} E\left(\frac{2 \sqrt{r\left(1-\varepsilon \rho^{\prime}\right)}}{r+1-\varepsilon \rho^{\prime}}\right)\left[\tilde{b}_{0}(\rho)+\tilde{b}_{1}(\rho)\right] \\
& =-\int_{0}^{\infty} \frac{d \rho}{\left(\rho-\rho^{\prime}\right)^{2}} \tilde{b}_{1}(\rho)+\int_{1 / \varepsilon}^{\infty} \frac{d \rho}{\left(\rho-\rho^{\prime}\right)^{2}} \tilde{b}_{1}(\rho)-\int_{0}^{\infty} \frac{d \rho}{\left(\rho-\rho^{\prime}\right)^{2}} \tilde{b}_{0}(\rho) \\
& +\int_{1 / \varepsilon}^{\infty} \frac{d \rho}{\left(\rho-\rho^{\prime}\right)^{2}} \tilde{b}_{0}(\rho)-\frac{\varepsilon}{2} b_{0} \ln \varepsilon-\frac{\varepsilon}{2} b_{0} \ln \rho^{\prime}-\frac{\varepsilon}{2} \int_{0}^{\infty} \frac{b_{0}-\tilde{b}_{0}(\rho)}{\rho-\rho^{\prime}} d \rho+o(\varepsilon)
\end{aligned}
$$

The second and fourth integrals on the right-hand side of (23) are calculated with the use of the asymptotic expansions (18), (22). The results are

$$
\int_{1 / \varepsilon}^{\infty} \frac{d \rho}{\left(\rho-\rho^{\prime}\right)^{2}} \tilde{b}_{0}(\rho)=\varepsilon b_{0}+o(\varepsilon), \quad \int_{1 / \varepsilon}^{\infty} \frac{d \rho}{\left(\rho-\rho^{\prime}\right)^{2}} \tilde{b}_{1}(\rho)=o(\varepsilon) .
$$

Thus, the problem for the boundary layer function $\tilde{b}_{1}\left(\rho^{\prime}\right)$ is

$$
\begin{array}{r}
-\int_{0}^{\infty} \frac{d \rho}{\left(\rho-\rho^{\prime}\right)^{2}} \tilde{b}_{1}(\rho)+\tilde{b}_{1}\left(\rho^{\prime}\right) \gamma^{\prime}\left(\tilde{b}_{0}\left(\rho^{\prime}\right)\right)=-\varepsilon b_{0}+\frac{\varepsilon b_{0}}{2}\left[\ln \varepsilon+\ln \rho^{\prime}\right] \\
+\frac{\varepsilon}{2} \int_{0}^{\infty} \frac{b_{0}-\tilde{b}_{0}(\rho)}{\rho-\rho^{\prime}} d \rho \\
\tilde{b}_{1}(0)=0, \quad \tilde{b}_{1}\left(\rho^{\prime}\right) \sim \frac{b_{0} \varepsilon}{\gamma^{\prime}\left(b_{0}\right)}\left[\frac{1}{2} \ln \varepsilon+\frac{1}{2} \ln \rho^{\prime}-1\right] \quad \text { as } \rho^{\prime} \rightarrow \infty
\end{array}
$$

Since Eq. (24) is linear, the solution $\tilde{b}_{1}\left(\rho^{\prime}\right)$ can be taken in the form

$$
\tilde{b}_{1}\left(\rho^{\prime}\right)=\varepsilon \ln \varepsilon \tilde{\phi}_{1}\left(\rho^{\prime}\right)+\varepsilon \tilde{\phi}_{2}\left(\rho^{\prime}\right) .
$$


The functions $\tilde{\phi}_{1}\left(\rho^{\prime}\right), \tilde{\phi}_{2}\left(\rho^{\prime}\right)$ are required to satisfy the problems

$$
\begin{gathered}
-\int_{0}^{\infty} \frac{d \rho}{\left(\rho-\rho^{\prime}\right)^{2}} \tilde{\phi}_{1}(\rho)+\tilde{\phi}_{1}\left(\rho^{\prime}\right) \gamma^{\prime}\left(\tilde{b}_{0}\left(\rho^{\prime}\right)\right)=\frac{b_{0}}{2} \\
\tilde{\phi}_{1}(0)=0, \quad \tilde{\phi}_{1}\left(\rho^{\prime}\right) \sim \frac{b_{0}}{2 \gamma^{\prime}\left(b_{0}\right)} \quad \text { as } \rho^{\prime} \rightarrow \infty \\
-\int_{0}^{\infty} \frac{d \rho}{\left(\rho-\rho^{\prime}\right)^{2}} \tilde{\phi}_{2}(\rho)+\tilde{\phi}_{2}\left(\rho^{\prime}\right) \gamma^{\prime}\left(\tilde{b}_{0}\left(\rho^{\prime}\right)\right)=-b_{0}+\frac{b_{0}}{2} \ln \rho^{\prime} \\
\quad+\frac{1}{2} \int_{0}^{\infty} \frac{b_{0}-\tilde{b}_{0}(\rho)}{\rho-\rho^{\prime}} d \rho \\
\tilde{\phi}_{2}(0)=0, \quad \tilde{\phi}_{2}\left(\rho^{\prime}\right) \sim \frac{b_{0}}{2 \gamma^{\prime}\left(b_{0}\right)} \ln \rho^{\prime}-\frac{b_{0}}{\gamma^{\prime}\left(b_{0}\right)} \quad \text { as } \rho^{\prime} \rightarrow \infty
\end{gathered}
$$

It should be observed that, in contrast with (26), the boundary layer solution in the case of a two-dimensional crack does not depend on $\ln \varepsilon$; it corresponds to the problem (27). This problem has been solved explicitly in [3] for the case of the linear function $\gamma$ and numerically in [4] for the case of the quadratic force law. (Here we do not present the results of those calculations and refer the reader to the papers [3], [4].)

Due to the presence of the function $\tilde{b}_{0}\left(\rho^{\prime}\right)$ on the right-hand side of Eq. (28), we solve the problem (28) numerically. The plots of $\tilde{\phi}_{2}$ for linear and quadratic functions $\gamma$ are shown, with $\sigma=1$, in Fig. 1 and Fig. 2, respectively.

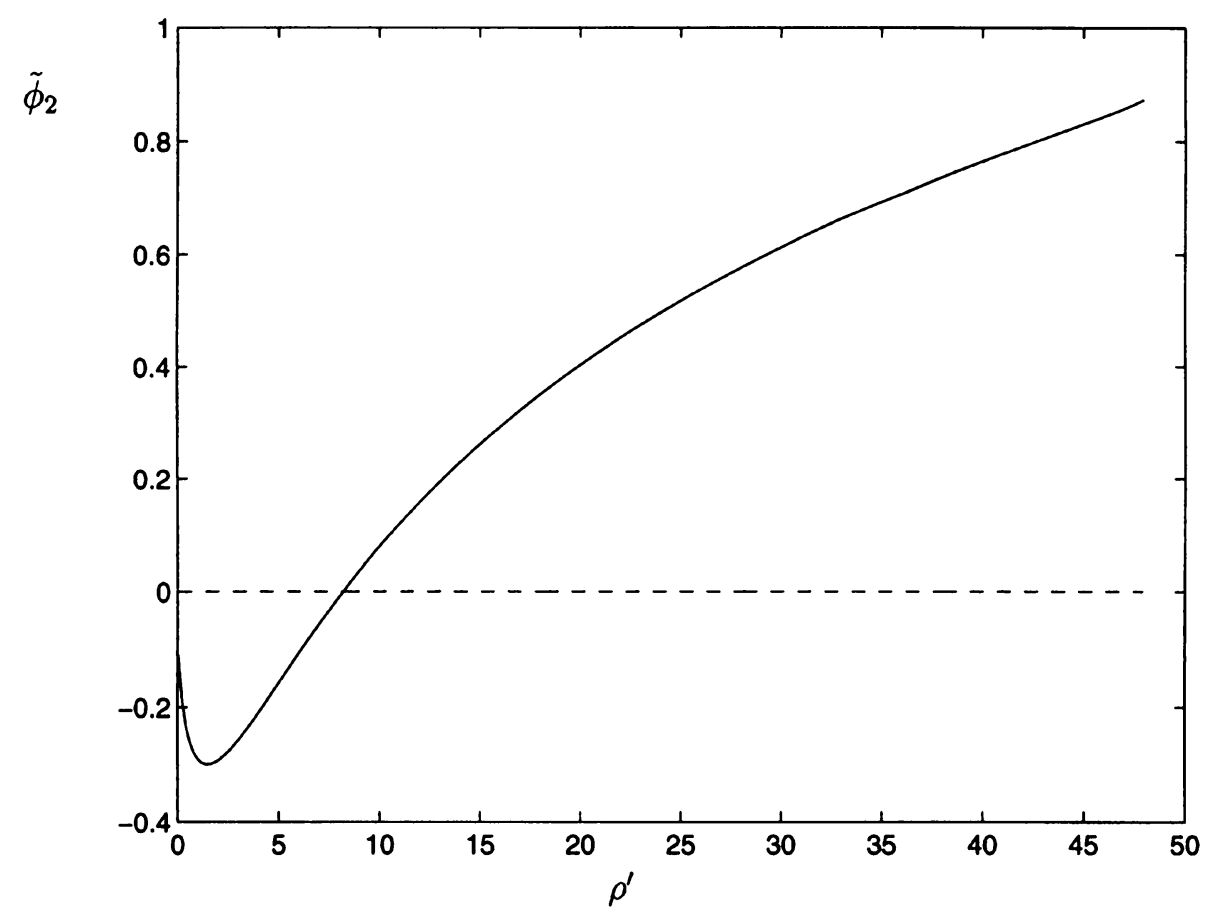

Fig. 1. Plot of the function $\tilde{\phi}_{2}$ for the linear force law $\gamma=b$ and $\sigma=1$ 


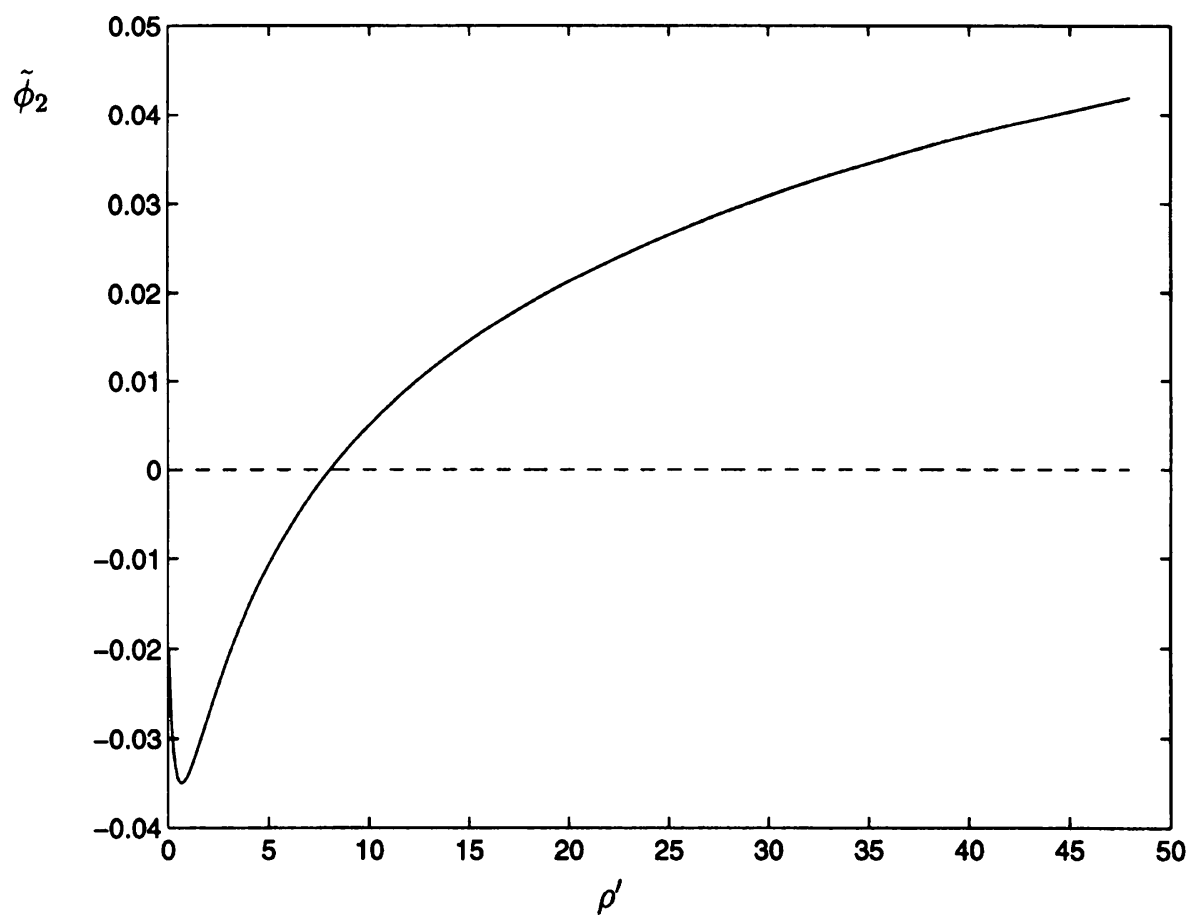

FIG. 2. Plot of the function $\tilde{\phi}_{2}$ for the quadratic force law $\gamma=$ $b\left(b_{*}-b\right)$ and $\sigma=1, b_{*}=5$

Stress intensity factor. Since only the boundary layer terms have the square root behaviour near the edge of the crack, the stress intensity factor is specified by the boundary layers. It has the form

$$
K_{I} \sim(2 / \pi)^{1 / 2}\left[K^{(0)}+\varepsilon \ln \varepsilon K^{(1)}+\varepsilon K^{(2)}+o(\varepsilon)\right] .
$$

Here,

$$
\begin{aligned}
& K^{(0)}=\int_{0}^{\infty} \frac{d \rho^{\prime}}{\sqrt{\rho^{\prime}}}\left[\sigma-\gamma\left(\tilde{b}_{0}\left(\rho^{\prime}\right)\right)\right], \\
& K^{(1)}=\int_{0}^{\infty} \frac{d \rho^{\prime}}{\sqrt{\rho^{\prime}}}\left[\frac{b_{0}}{2}-\tilde{\phi}_{1}\left(\rho^{\prime}\right) \gamma^{\prime}\left(\tilde{b}_{0}\left(\rho^{\prime}\right)\right)\right], \\
& K^{(2)}=\int_{0}^{\infty} \frac{d \rho^{\prime}}{\sqrt{\rho^{\prime}}}\left[-b_{0}+\frac{b_{0}}{2} \ln \rho^{\prime}+\frac{1}{2} \int_{0}^{\infty} \frac{b_{0}-\tilde{b}_{0}(\rho)}{\rho-\rho^{\prime}} d \rho-\tilde{\phi}_{2}\left(\rho^{\prime}\right) \gamma^{\prime}\left(\tilde{b}_{0}\left(\rho^{\prime}\right)\right)\right] .
\end{aligned}
$$

Note that in comparison with the case of a plane strain semi-infinite crack the asymptotic formula (29) gives the same principal term $K^{(0)}$, but it differs in the next terms. A plot of the factor $K^{(0)}$ is shown in Fig. 3 (see p. 336); this is the same as in [4]. The calculation of the coefficient $K^{(1)}$ follows the same pattern as in [3], [4]. For the linear case $(\gamma=k b$, 


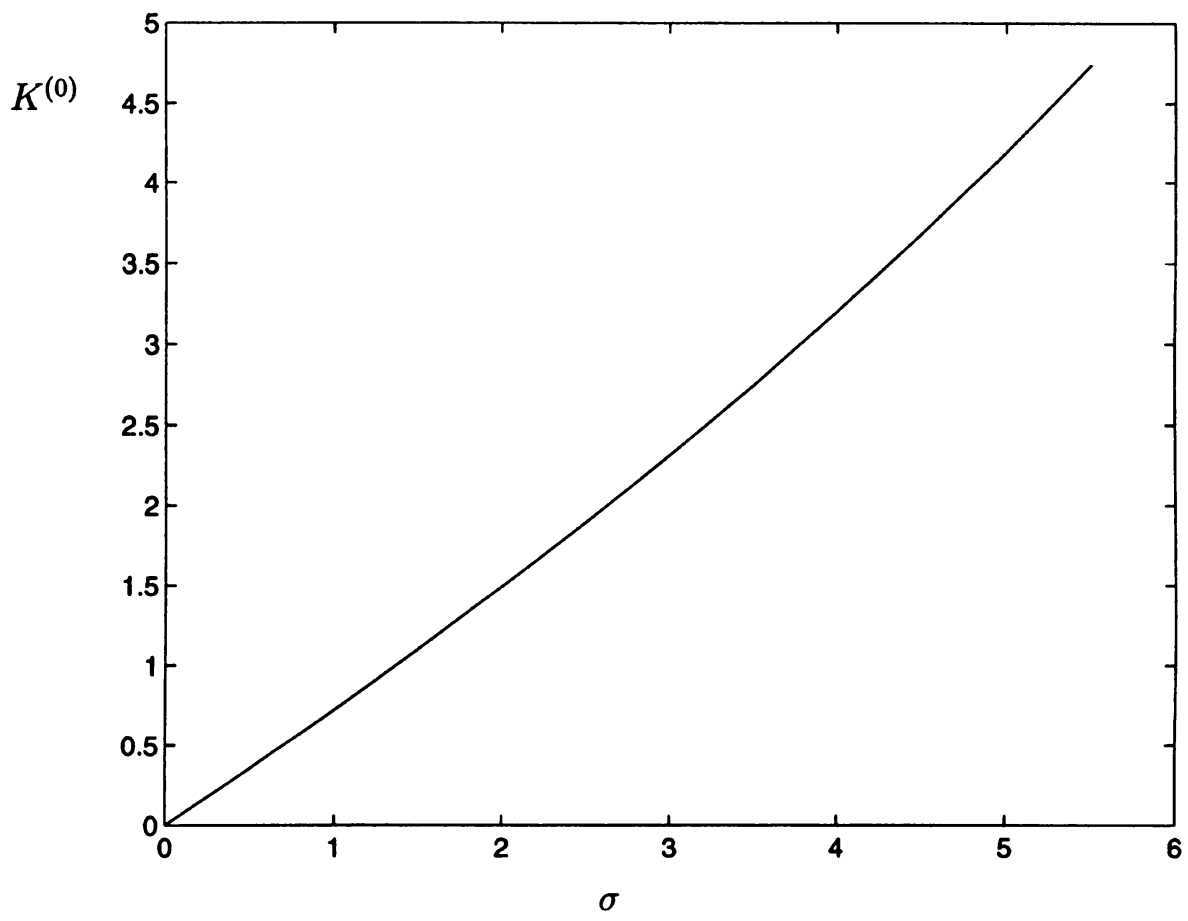

FIG. 3. $K^{(0)}$ versus $\sigma$, with $\gamma=b\left(b_{*}-b\right)$ and $b_{*}=5$

$k$ is a positive constant) the factor $K^{(1)}$ is specified by

$$
K^{(1)}=\frac{\pi}{4} k^{-3 / 2} \sigma .
$$

The equality (33) follows from the representation of the solution $\tilde{\phi}_{1}\left(\rho^{\prime}\right)$ of the problem (27) obtained in [10]

$$
\begin{gathered}
\tilde{\phi}_{1}\left(\rho^{\prime}\right)=\frac{b_{0}}{2 k} V\left(\frac{k \rho^{\prime}}{\pi}\right), \\
V(\eta)=\int_{0}^{\eta} v(\xi) d \xi=1-\int_{\eta}^{\infty} v(\xi) d \xi \\
v(\eta)=\frac{1}{\pi} \int_{0}^{\infty} \frac{t d t}{\left(1+t^{2}\right)^{3 / 4}} \exp \left[-\eta t-\frac{1}{\pi} \int_{0}^{t} \frac{\ln r d r}{1+r^{2}}\right],
\end{gathered}
$$

and from the asymptotic expansion (see [10])

$$
V(\eta) \sim 2 \pi^{-1 / 2} \eta^{1 / 2} \text { as } \eta \rightarrow 0
$$

For the quadratic case $\left(\gamma=k b\left(b_{*}-b\right), k\right.$ and $b_{*}$ are positive constants) the factor $K^{(1)}$ is evaluated from (31). The coefficient $K^{(1)}$ versus the load $\sigma$ is presented in Fig. 4; one can see that $\varepsilon \ln \varepsilon K^{(1)}<0$. The quantity $K^{(2)}$ is plotted in Figs. 5 and 6 (see p. 338) for the linear and quadratic function $\gamma$, respectively. They show that the coefficient $K^{(2)}$ is negative. 


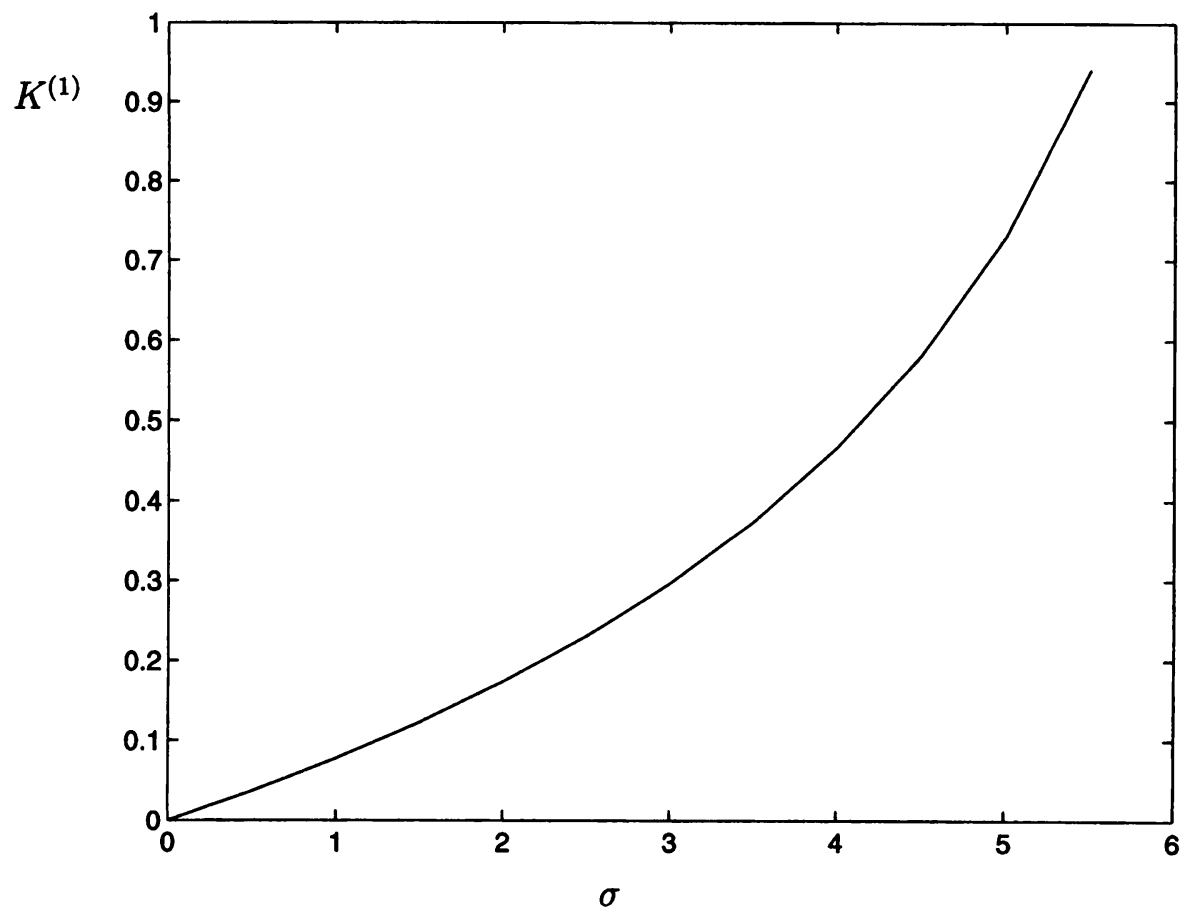

FIG. 4. $K^{(1)}$ versus $\sigma$, with $\gamma=b\left(b_{*}-b\right)$ and $b_{*}=5$

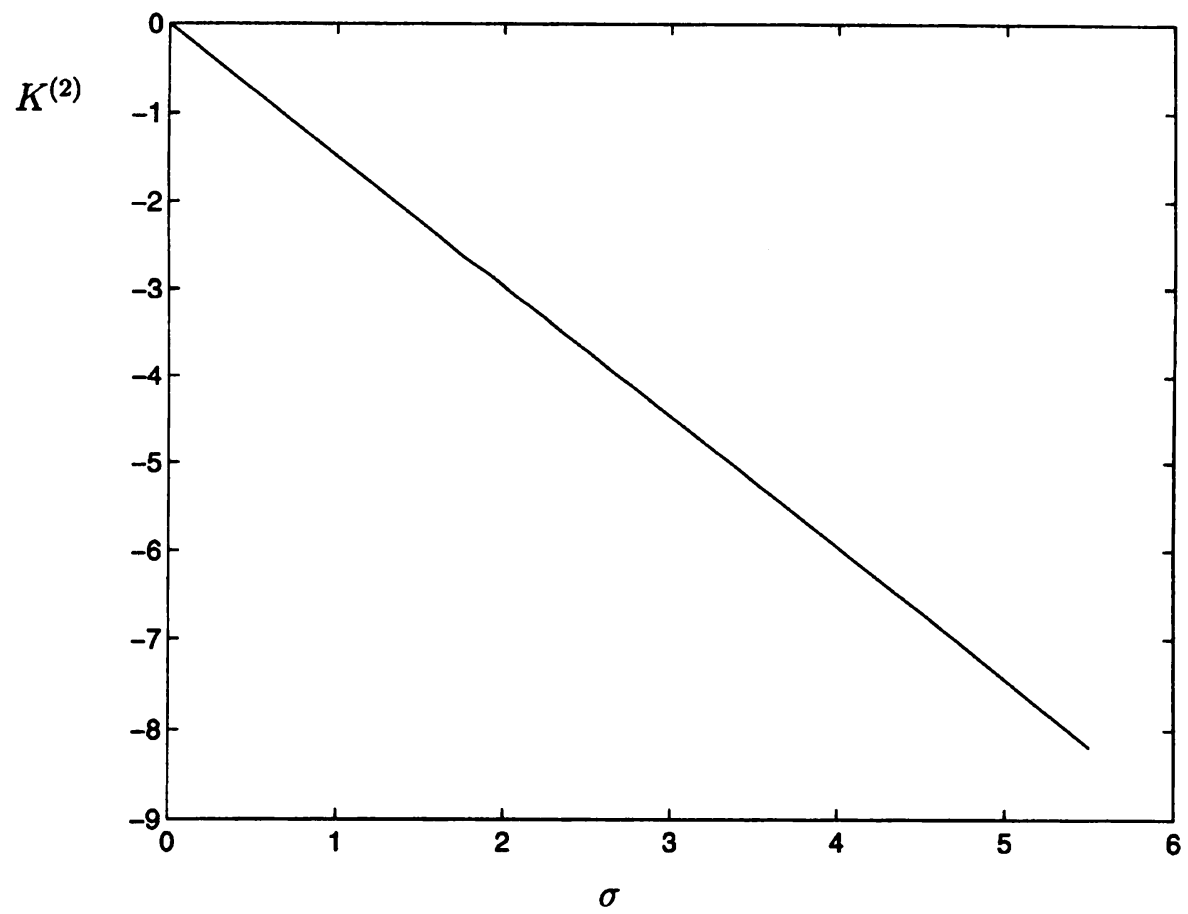

FiG. 5. $K^{(2)}$ versus $\sigma$, with $\gamma=b$ 


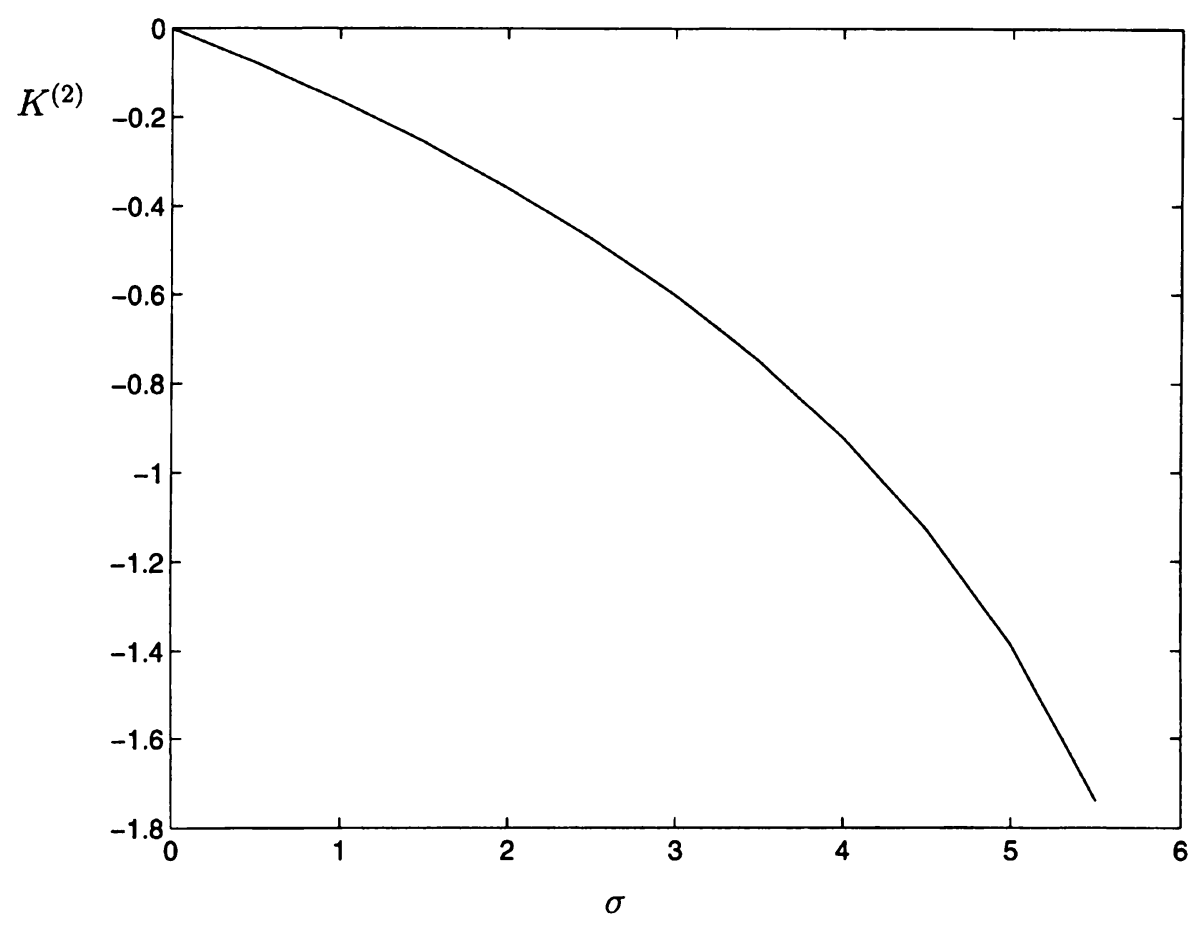

FIG. 6. $K^{(2)}$ versus $\sigma$, with $\gamma=b\left(b_{*}-b\right)$ and $b_{*}=5$

Evaluation of the critical load. Suppose $\gamma(b)$ is a smooth function and takes its maximum value at $b=b_{m}$. Then, $\gamma^{\prime}\left(b_{m}\right)=0$. Suppose, also, that the crack will extend if the stress intensity factor $K_{I}$ reaches a critical value $K_{c}$. The load $\sigma$ can become critical in one of two ways. The stress intensity factor at the crack edge may reach its critical value $K_{c}$ before the maximum value $b_{m}$ of $b$ is reached. If this happens, the crack will extend and, as it does so, $K_{I}$ will increase further. The crack opening displacement will also increase until, at the crack centre, $b=b_{m}$. Thereafter, a region of fibre breakage will expand from $r^{\prime}=0$, inducing further increase of $K_{I}$. Alternatively, the crack opening displacement $b$ may reach the critical value $b_{m}$ at $r^{\prime}=0$, while the stress intensity factor $K_{I}$ is below $K_{c}$. Fibres near $r^{\prime}=0$ will break, thereby allowing further crack opening as well as increase of $K_{I}$. Either event will precipitate instability and therefore rapid crack growth, so long as the load $\sigma$ is maintained.

Here, the critical load for the attainment of $b_{m}$ is estimated, assuming that condition $K_{I}<K_{c}$ is maintained. We suppose that the load

$$
\sigma=\sigma_{m}+\sigma_{1}
$$

with $\sigma_{m}=\gamma\left(b_{m}\right)$ and $\sigma_{1}$ being small, is applied on the crack faces, and the relative displacement at the centre of the crack is

$$
b(0)=b_{m} .
$$

The objective is to estimate the value of the small correction term $\sigma_{1}$ that achieves the condition (35). 
The solution to lowest order of the problem (6), (7) with $\sigma$ given by (34) is specified by

$$
b\left(r^{\prime}\right) \sim b_{m}+\tilde{b}_{0}\left(\rho^{\prime}\right)-S_{0}^{0}\left(\rho^{\prime}\right)+o(1),
$$

where $S_{0}^{0}\left(\rho^{\prime}\right)=b_{m}$. The boundary layer function $\tilde{b}_{0}\left(\rho^{\prime}\right)$ satisfies the problem

$$
\begin{gathered}
-\int_{0}^{\infty} \frac{d \rho}{\left(\rho-\rho^{\prime}\right)^{2}} \tilde{b}_{0}(\rho)+\gamma\left(\tilde{b}_{0}\left(\rho^{\prime}\right)\right)=\sigma_{m}, \\
\tilde{b}_{0}(0)=0, \quad \tilde{b}_{0}\left(\rho^{\prime}\right) \sim b_{m} \quad \text { as } \rho^{\prime} \rightarrow \infty .
\end{gathered}
$$

As $\varepsilon \rightarrow 0$, the asymptotic expansion of $\tilde{b}_{0}\left(\frac{1-r^{\prime}}{\varepsilon}\right)$ has the form

$$
\tilde{b}_{0}\left(\frac{1-r^{\prime}}{\varepsilon}\right) \sim b_{m}-\left[-\frac{2 b_{m}}{\gamma^{\prime \prime}\left(b_{m}\right)}\right]^{1 / 2} \sqrt{\frac{\varepsilon}{1-r^{\prime}}}+o(\sqrt{\varepsilon}) \quad \text { as } \varepsilon \rightarrow 0 .
$$

The function $b_{1}\left(r^{\prime}\right)$ solves the equation

$$
-\varepsilon \int_{0}^{1} \frac{d r}{\left(r-r^{\prime}\right)^{2}} \frac{2 r}{r+r^{\prime}} E\left(\frac{2 \sqrt{r r^{\prime}}}{r+r^{\prime}}\right) b_{m}+\frac{1}{2} \gamma^{\prime \prime}\left(b_{m}\right) b_{1}\left(r^{\prime}\right)^{2}=\sigma_{1},
$$

with fixed $r^{\prime} \in(0,1)$. It follows from (8) that

$$
b_{1}\left(r^{\prime}\right)=-\left[-\frac{2}{\gamma^{\prime \prime}\left(b_{m}\right)}\right]^{1 / 2}\left[-\sigma_{1}+\varepsilon b_{m}\left\{\frac{1}{1-r^{\prime}} E\left(\frac{2 \sqrt{r^{\prime}}}{1+r^{\prime}}\right)+\frac{1}{1+r^{\prime}} K\left(\frac{2 \sqrt{r^{\prime}}}{1+r^{\prime}}\right)\right\}\right]^{1 / 2},
$$

and therefore,

$$
b_{1}\left(r^{\prime}\right) \sim-\left[-\frac{2}{\gamma^{\prime \prime}\left(b_{m}\right)}\right]^{1 / 2}\left[-\sigma_{1}+\varepsilon \pi b_{m}\right]^{1 / 2} \quad \text { as } r^{\prime} \rightarrow 0
$$

The condition (35) then gives

$$
\sigma_{1}=\varepsilon \pi b_{m}
$$

Thus, the critical value of the applied load $\sigma$ is specified by

$$
\sigma_{\text {crit }} \sim \sigma_{m}+\varepsilon \pi b_{m}+o(\varepsilon) \text { as } \varepsilon \rightarrow 0 .
$$

In dimensional variables (4)-(5) the relation (40) has the form

$$
\bar{\sigma}_{\text {crit }} \sim \bar{\sigma}_{m}+\frac{\mu(\mu+\lambda)}{2(\lambda+2 \mu)} \frac{\bar{b}_{m}}{a}+o(1 / a) \quad \text { as } a \rightarrow \infty .
$$

Note that the asymptotic representation for $\sigma_{\text {crit }}$ in the case of a two-dimensional crack was obtained in [7] (p. 96, formula (19)). The relations (40) and (19) of [7] give the same principal term $\sigma_{m}$, but they are different in the terms of order $O(\varepsilon)$.

The principal term $K^{(0)}$ of the stress intensity factor $K_{I}$ at the edge of the crack is given by (30), with $\sigma=\sigma_{\text {crit }}$ and $\tilde{b}_{0}$ specified by (36)-(38). The results of numerical calculation presented in Fig. 3 show that for the quadratic function $\gamma$ the factor $K^{(0)}$ monotonically increases with increasing $\sigma$, and therefore, the condition $K^{(0)}<K_{c}$ implies the relation $\sigma_{\text {crit }}<\sigma_{k}$, where $\sigma_{k}$ denotes the value of the applied load that provides the condition $K_{I}=K_{c}$. 
Acknowledgment. Support from the Engineering and Physical Sciences Research Council (Grant GR/H54140) is gratefully acknowledged.

\section{REFERENCES}

[1] D. B. Marshall and B. N. Cox, Tensile fracture of brittle matrix composites: Influence of fibre strength, Acta Metall. 35, 2607-2619 (1987)

[2] L. N. McCartney, Mechanics of matrix cracking in brittle-matrix fibre-reinforced composites, Proc. Royal Soc. London A409, 329-350 (1987)

[3] J. R. Willis and S. Nemat-Nasser, Singular perturbation solution of a class of singular integral equations, Quart. Appl. Math. 48, 741-753 (1990)

[4] A. B. Movchan and J. R. Willis, Asymptotic analysis of the reinforcement of a brittle crack by bridging fibres, Quart. J. Mech. Appl. Math. 46, 331-350 (1993)

[5] J. T. Guidera and R. W. Lardner, Penny-shaped cracks, Journal of Elasticity 5, 59-73 (1975)

[6] J. Hadamard, Lectures on Cauchy's Problem in Linear Partial Differential Equations, Dover, New York, 1952

[7] J. R. Willis, Asymptotic analysis of crack bridging by ductile fibres, Composites 24, 93-97 (1993)

[8] A. B. Movchan and J. R. Willis, An asymptotic procedure and numerical study for the analysis of an elastic body with a thin subsurface crack, Euro. J. Appl. Math. 6, 1-23 (1995)

[9] M. D. Van Dyke, Perturbation Methods in Fluid Mechanics, Academic Press, New York, 1964

[10] W. E. Olmstead and A. K. Gautesen, Asymptotic solution of some singularly perturbed Fredholm equations, Z. Angew. Math. Phys. 40, 230-244 (1989) 